\title{
III.
}

\section{Die Ganglien in den harnabführenden Wegen des Menschen und einiger Thiere.}

Von Professor Rud. Maier in Freiburg.

(Hierzu Taf. II - III.)

Die Nachforschungen nach den kleineren mikroskopischen Nervenknötchen, welche in den peripherischen Ausbreitungen des Nervensystems in einer Reihe von Geweben und Organen sich vorfinden, sind, seit den ersten Anregungen, die Remak's Untersuchungen dieser Frage gegeben, nicht mehr wieder zur Ruhe gekommen. Sie dehnten sich von den zuerst in Angriff genommenen oder von den nur flüchtig tangirten Objecten allmäblich und in eingehenderer Weise auf fast sämmtliche Organe aus, in welchen solche Vorkommnisse vermuthet werden konnten. Von den Arbeiten über die einzelnen Fundstätten werde ich natürlich nur diejenigen aufführen, welche mein hier behandeltes Thema beruhren und auch diese so kurz wie möglich, da die betreffende Literatur in erschöpfender Weise in den grossen Werken von Kölliker, Henle und Frey neben den eigenen Angaben dieser Autoren zusammengetragen sich findet. Einzelne Capitel davon sind Theile allgemeiner grosser Fragen, wie die Endigungen der Nerven in den Schleimhäuten und in dem Muskelgewebe. Diese ubergehe ich ganz. Ebenso die Untersuchungen über die Nerven und Ganglien der Respirationswege, der Thränen- und Speicheldrüsen, der Generationsorgane und des Gefässsystems. Die über den Digestionstractus werde ich nur insofern hier kurz beruhren als in diesen Arbeiten zum Theil auch solche uber die Harnorgane niedergelegt sind.

Die ersten Arbeiten darüber legte Remak in einer Reibe von Aufsätzen vom Jahre $1840-\mathbf{5 8}$ der medicinischen Welt vor. Seine Untersuchungen erstreckten sich in der ersten dieser Arbeiten (1840) auf die Ganglien der Zunge und des Schlundes beim Rinde. Im Jahre 1847 folgte seine Publication uber ein selbständiges Darm- 
nervensystem (besonders Vögel). Im Jahre 1852 fügte er die Wand der Harnblase des Schweins, die Wand des Magens bei Wirbelthieren hinzu, dann die Zunge bei Menschen und Säugethieren und 1858 beschrieb er wieder die peripherischen Ganglien an den Nerven des Nahrungsrobres. - Im Jahre 1857 machte Meissner seine vorläufige Mittheilung über die Ganglien des Darmes. Seine Angaben galten für den Darm des Menschen, des Schweins und Rindes und sind durch diese Ausdehnung auf die Säugethiere und da sie zum ersten Male die betreffenden Partien auch beim Menschen in die Untersuchung hereinzogen, besonders wichtig und für die Ganglienapparate des Darmes grundlegend. - Im Jahre 1858 veröffentlichte Billroth seine Arbeiten uber die Schlundschleimhaut (Wassersalamander, Frosch, Schildkröte), Rachen (Kaninchen), den Magen (Frosch, Wassersalamander), den Dünndarm (beim Kind) und die Harnblase (Frosch, Schildkröte). Darauf folgte Manz (1859) mit seinem Aufsatze ïber die Ganglien und Nerven des Darms. Er rückte die Untersuchung der Darmganglien in weitere Gebiete vor (Schwein, Kalb, Ochse, Kaninchen, erwachsener Mensch und Kind) und fügte diesen noch die Beschreibung der Harnblasenganglien des Frosches bei und der Vorhofsganglien bei demselben -Thier. Er beherrschte so ein reicheres Material als seine Vorgänger und es sind deshalb die Ergebnisse seiner Arbeit von besonderer Wichtigkeit. Allejn er hat auch eingehender als das bis dahin geschehen, seine mikroskopischen Untersuchungen auf einzelne bis dahin noch nicht sicher ermittelte Punkte gerichtet. Dahin sind zu rechnen das Verhalten der Hüllen der Ganglien, die Bedeutung der verschiedenen hier beobachteten Kerngebilde, die Natur der Nervenfasern und das Verhalten der Ganglienzellen bezüglich jhrer Fortsätze. Er fand nur unipolare Zellen oder wenn mehrere Ausläufer vorhanden waren, gingen sie alle nach einer Richtung. Meissner dagegen hat bipolare beschrieben. Von den diesen Arbeiten vächstfolgenden Untersucbungen sind noch zu nennen die von Breiter (1861), Frey (1862) und Auerbach, der im Jahre 1862 und später 1864 den bisher beschriebenen Ganglien der Submucosa des Darmes noch die analogen Gebilde in Form eines Plexus myentericus hinzufügte. Weitere Ausführungen über diese Gebilde verdanken wir dann noch den Untersuchungen von Gerlach (1873), Klein (1873) und neuestens Drasch (1880). 
Ueber die Harnorgane besitzen wir, wie schon erwähnt, die ersten Angaben wieder von Remak aus dem Jahre 1847 uber Nerven der Ureteren der Vögel und aus dem Jahre 1852 uber die Nerven und Ganglien der Harnblase vom Schwein. Daran schlossen sich die ebenfalls schon erwähnten Angaben von $\mathrm{Manz}$ (1858) uber die Harnblase des Frosches, denen er im Jahre 1860 seine Untersuchungen über die Nervenausbreitung im Ureter der Vögel hinzufugte. His beschrieb Nervenendigungen in der Harnblase des Hundes (1863) und Klebs (1863 und 1865) in eingehender Weise das Verhalten der Nerven zar Muscularis der Blase vom Frosche. Kisselew (1868) behandelte die Frage über die Endigung der sensiblen Nerven in der Harnblase des Frosches und Lardowsky 1871, 72) das gleiche Thema mit der Ausdehnung auf die gleiche Frage auch bezuglich der Musculatur.

Es ist somit bei verschiedenen Thieren das Vorkommen von Ganglien in der Wand der Harnblase und des Ureters constatirt worden. In diesem Stadium griff ich das Thema wieder auf, da ich anlässlich der Untersuchung von Darmganglien aus andern Gründen in die Lage kam, auch die Harnblase versehiedener Thiere auf die gleichen Gebilde zu untersuchen. Das führte auf den Gedanken, die Nachforschungen auf den gesammten harnabfuhrenden Apparat auch beim Menschen auszudehnen. Ich beauftragte daher, da berufliche Geschäfte mir zunächst die Untersuchung nicht gestatteten, einen Schüler von mir, Le o Köhler, in meinem Institut bei verschiedenen Thieren und auch beim Menschen Untersuchungen uber die Harnblase in der Richtung anzustellen. Er kam diesem Auftrag nach und wir konnten bald die fruheren Angaben uber die Ganglien der Harnblase des Frosches bestätigen. Dann folgten die ähnlichen Befunde in der Harnblase des Kaninchens und endlich des erwachsenen Menschen. Weiter konute Herr Köhler, der diese Untersuchung zu seiner Dissertation benutzte, die Sache nicht verfolgen, da er von der Universităt abging. Ich unterzog mich in Folge dessen, um die Arbeit in der vorgesetzten ganzen Ausdehnung zum Abschluss zu bringen, der weiteren Nachforschung. Was die Art der Untersuchung betrifft, so bin ich nach verschiedentlichem Durchprobiren, Verlassen und modificirter Wiederaufnahme von mancherlei Methoden sowohl der Härtung (Chromsäure, Acidum tannicum) als der Quellung und Maceration (Essigsäure, 
verdünnte Schwefelsäure) sowie der Färbung (Chlorgold, Goldchloridkalium, Chlorgold, Palladium, Pikrinsäure u. s. w.) schliesslich wieder zu dem einfachsten zurückgekehrt. Ich legte die zu späteren Schnitten oder zum Zerzupfen bestimmten Stücke zuerst in schwachen Holzessig und liess sie verschiedentlich lang darin, kürzer, wenn ich sie schneiden, länger, wenn ich die einzelnen Lagen nur schichtenweise abtragen wollte. Letzteres fand ich, wie auch schon Manz es bemerkte, als das Tauglichste, indem von dem gequollenen Präparate, wenn es erst die richtige Weichbeit erhalten hatte, mit der Pincette, von der Oberfläche in die Tiefe gehend, beliebig grosse dlinne Platten schichtenweise abgezogen wurden. Da das bei der gebörigen Maceration sehr leicht ging, so geschah es ohne jedes gröbere Insult des Gewebes. Schnitte mit dem Messer oder der Scheere, wagerecht oder senkrecht geführt, erwiesen sich als weniger brauchbar. Ohne jede Färbung, nur mit Wasser und Glycerin oder mit Serum befeuchtet, wurden die dunnen abgezogenen Häutchen, die äusserst leicht und ohne jede Zerrung und Dehnung sich ablösen liessen, der mikroskopischen Untersuchung unterzogen. So auch konnten viel sicherer die einzelnen Lagen der Mucosa, Submucosa, Muscularis nach einander durchforscht und die successiven Verhältnisse und etwaige Veränderungen constatirt werden. Einfache Maceration in Salzwasser und spätere Anwendung von Chlorgold $(0,01 \mathrm{pCt}$.) gab auch gute Präparate, aber nie so brauchbare für die nähere Untersuchung des Details. Die spätere Anwendung einer concentrirten Lösung von Acidum tannicum unter Einwirkung von hoher Temperatur babe ich nie sehr brauchbar gefunden, ebenso wenig wie die Anwendung von Carmin mich so sehr in der Untersuchung gefördert hätte. Meine Untersuchungen haben sich auf die ganze Ausdehnung der harnabführenden Wege erstreckt und ich muss als Resultat derselben die Angabe machen, dass soweit die Schleimhautausbreitung und eine sie umfassende Muskelscbicht reicht also hinauf bis zu den Markpapillen und hinab bis zur Pars bulbosa der Harnröhre sich die fraglichen Ganglien vorfinden und zwar nicht nur beim Thier (Säugethiere, Frosch) sondern auch beim Menschen. Ueberall treten im Verlaufe der Nerven in der Mucosa und Muscularis Ganglienzellen auf und verleihen diesen Ausbreitungen dadurch einen ganz bestimmten Charakter, ganz ähnlich den von Remak, Meissner, 
Manz, Auerbach beschriebenen gangliösen Apparaten der Darmwand des Menschen und der Thiere. Die Art, der zum Theil dem Willen entzogenen Muskelbewegungen dieser Theile, die Periodicität und die wellenförmige von gewissen Punkten einsetzenden und nach gewissen Richtungen sich fortpflanzenden Bewegungen liessen dies von vornherein erwarten. Auch die Harnabfuhrwege sind in ihrer ganzen Ausdehnung, und nun auch für den Menschen giltig, anatomisch in den Kreis der Organe eingetreten, welche nicht ausschliesslich von gewissen in das Cerebrospinalsystem gelegten Centren des Sympathicus aus innervirt werden, sondern wie das Herz und der Darm automatische Apparate besitzen und so in directester Weise Reize in Form von Muskelbewegungen auszulösen im Stande sind.

Ueberall sind die Ganglien den Nervenbahnen der Mucosa und Muscularis eingeschaltet und unterscheiden sich theils nur durch den hauptsächlichsten Sitz je nach den verschiedenen anatomischen Verhältnissen der einzelnen Stationen, theils nur durch Form, Grösse und allgemeine Anordnung der Zellen selbst und der ihṇen anliegenden Nervenfasern. Als einfachste Form ist das Verbåltniss anzuführen, dass in den Verlauf einer einzelnen Nervenfaser sich eine oder mehrere hinter einander gelegene Zellen eingelagert finden. Das Neurilem der Fasern baucht sich an der betreffenden Stelle aus und es bildet sich so eine grossvaricöse oft im Verlaufe der Faser roserkranzförmig sich wiederholende Anschwellung derselben (Taf. III. Fig. 11, 16). Es sind meist blasse Fasern und wenn die Hülle derselben nicht sebr deutlich ist und die Faser sehr blass, scheint der Körper der Faser von Stelle zu Stelle grössere rundliche Anschwellungen zu haben. Immer aber lässt sich ein deutlicher Kern, umgeben von feiner Granulirung, an der Stelle wahrnehmen. Ist die Hülle deutlich, namentlich durch Kerne sehr markirt, siebt man dieselbe über die kleinen Hügel hinweggehen und die Contouren der Zelle treten dann auch oben und unten, soweit sie der Hülle parallel gehen, deutlicher hervor. Da wo die Zelle innerhalb der Hülle der Nervenfaser an den Inbalt derselben grenzt, gehen beide unmittelbar und ununterbrochen in einander uber, oder man trifft auch seitliche Contouren, wie wenn die Zelle auch hier eine Ausbuchtung der Faser bildete. Immer geht die Faser in die Substanz der Zelle uber und bildet so gleichsam Zu- 
und Abgang, also eine bipolare Zelle. Das ist auch die einzige Art von bipolaren Zellen, die ich sah. Nicht selten sind im Verlaufe eines solchen Nerven auch noch kleinere zellige Gebilde neben diesen grösseren deutlich ganglionären eingeschoben, welche sich in der Grösse oft wenig von den Kerngebilden des Neurilems unterscheiden. Nur ist oft neben durch Färbungen deutlich zu markirenden Kernen auch noch etwas mehr umgebende Protoplasmamasse zu erkennen und nicht selten zeigen auch sie feine Granulirung derselben. Sie sind offenbar als schwach entwickelte Formen der ersteren zu betrachten. - Eine zweite Form der Anordnung präsentirt sich als grössere Anbäufung von Ganglienzellen an den Stellen von Theilungen oder Kreuzungen oder Durchflechtung von Nervenfasern. Solche Nervenplexus und Abzweigungsstationen scheinen mitunter förmlich übersät und durchsetzt von Ganglienzellen, bald einzeln aber in grossen Mengen zerstreut zwischen den Nervenfasern eingelagert, bald in Gruppen beisammen liegend, grosse knotenförmige Gebilde inmitten dieser Durchflechtungen darstellend. Oft begleiten dann auch noch einzelne Zellen einzelne austretende Fasern in Form ersterer oben beschriebener Einlagerungsweise. Die Bilder sind daher sehr verschieden und zeigen sich wechselnd von den einfachsten spaltförmigen Einlagerungen $z$ wischen in ibrem parallelen Lauf auseinander tretenden und wieder sich aneinander legenden Nervenfasern bis zu der Bildung grösserer Knoten in Folge dicht aneinander gedrängter Nervenzellen, neben und über welchen die Nervenfasern wegziehen. Oft finden sich auch von der einen Seite herantretend wenige Züge verbundener Nervenfasern, gleichsam in einen solchen Plexus eintretend und dann auf der andern Seite zahlreicher und radienartig sich fortsetzend (Taf. II. Fig. 2), so dass es den Anschein hat, als ob aus diesem Ganglion entsprungene Fasern sich nun den andern beigesellt hätten. Oft auch bildet ein solcher Ganglienknoten den Mittelpunkt (Taf. II. Fig. 1) sich durchflechtender Fasern, von dem gleichmässig nach den verschiedensten Seiten bin Nervenfaserstränge abgehen. In den erwähnten Fällen haben die einzelnen Zellen keine Umhullung vom Neurilem der Nervenfasern, sondern nur von perineuritischem Bindegewebe, das kleinere mehr rundliche als spindelförmige Zellen oder Kernformen zeigt und ausserordentlich zartfaserig erscheint. Da wo die Nervenzellen dichter aneinander liegen, ist dasselbe bald 
körnig, bald homogen. - In einer dritten Form lagern grössere Gruppen von Zellen dem Zuge eines Nervenfasernbündels an. Es geschieht das bäufig in der Art, dass an der Seite der Anlagerung das den Nervenfaserzug umgebende Bindegewebe von diesem abgeht und sich ausbauchend als Hülle die Zellengruppe umfasst, um dann wieder jenseits an die Nervenfasern sich anzuschliessen. In solchen Fällen hat also das Ganglion auf der einen, innern, Seite die Nervenfasern, auf der andern, äussern, Seite eine bindegewebige Hülle als Begrenzung. So erscheint dann das Ganglion wie ein seitlicher Auswuchs am Nervenzweigè. In andern Fällen umgiebt ein Nervenfaserzug, an ein Ganglion herantretend, dasselbe je $\frac{2}{3}$ oder $\frac{8}{4}$ seines Umfangs im Halbbogen umgreifend, um dann wieder in der alten Richtung weiter zu ziehen. Hier hat dann das Ganglion seine eigene Hülle, die nur meist an der Stelle, wo es am Nerven anliegt, mit derjenigen des letzteren verschmilzt. Endlich findet sich das Bild auch noch so, dass das in die gemeinschaftliche Nervenhülle eingeschlossene Ganglion statt durch die Hülle fest an das Nervenbündel angeschlossen zu werden, sich von diesem enfernt, die gemeinschafliche Htille stielartig nachzieht und so bald beeren- bald traubenförmig seitlich an einer Nervenfaserbahn anhängend erscheint (Taf. II. Fig. 3). In allen diesen Fällen ist die Zellenfasergruppe von einer Hülle umschlossen. Doch darf man sich das nicht so vorstellen, dass ein selbständiger Schlauch, der wobl auch nach dem Ausfallen des Ganglion als leere Hülle zurückbliebe, das Ganze umgebe, sondern man kann nur constatiren, dass die Zellengruppen überall von einem Kerne tragenden Bindegewebe begrenzt erscheinen, welches an den Enden des Ganglion sich auf den Nervenfaserzug wieder fortsetzt. Ob man das also als eine Abtheilung des Perineurium ansehen, oder als eine selbständige mit der Bildung des Ganglion sich entwickelnde bindegewebige Scheide ansehen will, die sich mit dem alten Perineurium in Verbindung setzt, wird ziemlich gleichgiltig sein. Ueberall, wo grössere Gruppen von Zellen sich als geschlossenes Ganglion darstellen, findet sich ferner das Verhältniss so, dass einzelne kleinere Gruppen von Zellen von einer bindegewebigen Hulle umfasst und mehrere solche dann wieder durch eine weitere gemeinschaftliche umschlossen werden, so dass es eigentlich kejne allgemein gemeinschaftliche, sondern viele weitere und engere Hüllen giebt. Am 
besten wird man das thatsä chliche Verhältniss in der Art bezeichnen, dass das Perineurium an gewissen Stellen ein Maschengewebe bildet, in dessen Lücken die Zellen einzelue oder zu mehreren an Zahl eingelagert sich zeigen. Diese Anordnung, dass mehrere Zellen eine gemeinschaftliche Umbüllung besitzen und solche Gruppen dann wieder durch Bindegewebe zu grösseren Gruppen zusammengeschlossen werden, hat Manz bereits für die Ganglien des Darmes nachgewiesen. So besteht oft ein solcher Ganglienkörper aus secundären und tertiären Gruppen, die aneinander gelagert eben das ganze Ganglion ausmachen. Daher bilden einzelne Zellen mit ihrer Umhüllung selbst die Contouren eines solchen Ganglion und selten ist es und immer nur theilweise, dass noch eine weitere Umhüllung die äusserste Grenze schafft. Zwischen den einzelnen Zellen treten entweder eine oder mebrere Primitivfasern hindurch, oder es sind dieselben durch eine bald homogene, bald körnige Zwischensubstanz geschieden, etwa der Neurogliamasse der Gehirnrinde entsprechend. Immer finden sich in der nächsten Umgebung solcher Ganglien zablreiche Spindelzellen, welche oft bis dicht an die Grenze desselben herantreten und schliesslich mit den kleinen Spindel- oder Rundzellen des die Hulle umgebenden Bindegewebes eine continuirliche peripherische Zone nach aussen bilden. - Nicht selten sieht man, um auch dieser Figuren noch Erwähnung zu thun, einen nur wenige blasse oft bandartige Fasern umfassenden zarten Strang in eine knotenartige Verdickung endigen. In solchen Fällen kann man constatiren, dass entweder durch Einlagerung von Zellen in das Innere einer Primitivfaser, oder durch Anlagerung von Zellen an die Fasern die Verdickung entsteht. Das Bild erscheint dann ăhnlich den sog. Endknospen, oft wie eine birnförmige Anschwellung dieser Fasern, scheinbar ohne weitere Fortsetzung. Ich habe mich aber überzeugt, dass erstens in die Zusammensetzung dieser scheinbar terminalen Anschwellung immer mehrere Zellen eingehen und dann gewinnt man manchmal auch den Nachweis, dass jenseits dieses Knoten doch wieder Nervenfasern, wenn auch in anderer Richtung, weiter laufen. Spiralige Umschlingung dieser Fasern sah ich nie und es bedarf wohl keiner weitern Ausführung, um zu beweisen, dass es sich auch nicht um die von Beale und dann von J. Arnold genauer beschriebenen Endigung eines dunkelrandigen Nerven in eine Ganglienzelle handelt, wie solche aus dem 
Sympathicus des Frosches beschrieben worden sind und später als Nervenendigung auch in der Froschblase von Lardowsky aufgeführt wurden. Ich habe solche in der Schleimhaut der Blase weder beim Menschen noch beim Thiere gesehen.

Die meisten der mit einem Ganglion in Beziehung tretenden Nervenfasern sind blasse Fasern und nur seltener nehmen auch dunkelrandige in grösserer Anzahl daran Antheil. Letzteres geschieht immer nur in den äussern Wandschichten der Blase und des Ureter; in den innern Partien der Muskeln sowohl als der Schleimhaut stellen die blassen Fasern weitaus das grösste oder auch ausschliessliche Contingent. In den die genannten Organe aussen umhüllenden Bindegewebslagen kommen sogar nur breitere dunkelrandige Fasern vor und bilden hier grosse Durchflechtungen (sog. Grundplexus) mit auch eingelagerten Zellen. Ich habe die Zahl dieser Zellen nie sebr gross gefunden und rechne diese Bildungen eigentlich gar nicht zu den Ganglien, die ich hier beschreiben will. Die von diesen sich abzweigenden Fasern, welche in die Muscularis eintreten, sind meist feinere dunkelrandige und später gesellen sich zu diesen auch die blassen Fasern. Diese zusammen bilden die Hauptmasse der Geflechte in Muscularis und Mucosa und von diesen gehen dann wieder feinste varicöse dunkelrändige oder blasse bandartige Fasern ab, welche offenbar die Bedeutung von Endfasern haben. Die Beschreibung der Endigungsweise dieser liegt ausserhalb des Plans und der Absicht dieser Arbeit.

Die Zellen selbst sind wandungslos und zeigen ein blasses homogenes oder feinkörniges, manchmal auch grobkörniges Protoplasma mit deutlichem grossem Kern und Kernkörperchen, die nur hier und da durch grobkörnige Trübung des Zellenkörpers verdeckt sind. Manche Zellen zeichnen sich auch durch eine gewisse Färbung, namentlich leicht in's gelbliche oder bräunliche spielend, aus. Ihre Grösse wie ibre Form ist höchst wechselnd. Im Allgemeinen sind sie kleiner, als die im cerebro-spinalen Nervensystem eingelagerten. Es kommen solche von $0,02 \mathrm{Mm}$. und bis zu $0,001 \mathrm{Mm}$. herab vor. Bestimmte Grössenunterschiede $z$ wischen einzelnen Individuen, bei denen sie beobachtet worden, oder zwischen Species oder Genera, lassen sich nicht constatiren. Ihre Form ist bald rund, wo sie einzeln liegen, spindelförmig, länglich, wo sie in die Neurilemscbeide einer Primitivfaser eingezwängt sind oder poly- 
gonal, wo sie in Haufen gedrängt beisammen stehen. Es ist bei der Kleinheit dieser Zellen und andererseits dem Reichthum an oft sehr grossen anderen, dem Bindegewebe angehörigen Zellen und Kernen nicht selten schwierig zu entscheiden, ob man eine Ganglienzelle wirklich vor sich habe. Ich habe in solchen zweifelhaften Fällen das Verfahren eingeschlagen, dass ich solche Stellen nicht mit in die Beschreibung aufnahm. Nur wo ich Zellen fand ausgezeichnet durch ihr zartes homogenes Protoplasma, das gegenüber dem grossen Kern immer noch reich entwickelt sich zeigte, oder wo dieses durch feinere oder gröbere Körnung, namentlich zunächst dem Kern, oder allgemein eine gewisse Färbung erhielt oder gar deutlich gefärbt war, nur wo ich diese Zellen in unmittelbarer Verbindung mit unzweifelhaften Nervenfasern fand und wo diese Nervenfasern isolirt nicht nehen Gefässen verlaufend sich zeigten, habe ich die ganglionäre Natur des Gebildes angenommen. Da wo Fortsätze sich zeigten, war die Sache ohnehin entschieden.

Eine grosse Zahl der Zellen erscheint vollkommen isolirt, d. h. apolar, wenigstens lässt der dem Beschauer zugängliche Theil des Körpers keine Fortsätze abgehen. Ein anderer ebenfalls nicht geringer Theil dagegen erscheint mit einem solchen, zeigt sich unipolar. Auch hier muss ich, nach dem Vorgange Anderer, namentlich Manz, dessen Angaben über den allgemeinen Bau der Ganglien sowie speciell über den der Zellen selbst ich überall nur bestätigen kann, zwischen wahren und falschen Fortsätzen unterscheiden. Erstere sind wirkliche Fortsetzungen des Protoplasma nach irgend einer Seite hin und es zeichnen sich deshalb diese in der That durch ein gleich blasses, homogenes oder schwach körniges Aussehen ans, das keine Neurilemhülle zeigt und erst nach kürzerer oder längerer Zeit des Verlaufes sich mit einem Neurilem umgiebt (Taf. III. Fig. 8). Wenn die bindegewebige Umhüllung einer Zelle auch mit auf deren Fortsatz geht und später zu dessen Neurilem wird, da hält sie sich gerade an der Uebergangsstelle von Zelle in Fortsatz in einiger Entfernung von letzterem, gleichsam, wie das gut verglichen wurde, ein Dreieck bildend, dessen Basis von der Zelle eingenommen und dessen spitzer Winkel gegen die Nervenfaser gerichtet ist, in dessen Mitte der Fortsatz verläuft (Fig. 7 a). Dieser wahre Fortsatz reiht sich meist bald in parallelem Laufe anderen Nervenfasern an oder erst nach kürzerem selbstän- 
digem Verlauf, der oft nicht selten recht- oder spitzwinklig zu dem Nervenfaserzug steht, an den sich später die Faser anlegt. Es kommt dies selbstverständlich auf die Lagerung der Zelle an, von der der Fortsatz ausgeht, zu dem Nervenstrang, an den sich ersterer anlegt. So läuft der Fortsatz entweder direct aus der Zelle mit den anliegenden Fasern weiter, oder macht zuerst eine kleine winkelige Umbeugung, um dann erst mit den andern weiter zu zieheu. Anderemal läuft der Fortsatz spitzwinklig auf ein Nervenbündel zu und man kann ihn bei einigermassen breitem Nerven noch eine Strecke weit in dieser Transversalrichtung verfolgen, worauf er dann entweder plötzlich verschwindet oder an der gegenuberliegenden Seite angekommen, in der einen oder der andern Richtung weitergeht. An den Stellen, wo sich durchkreuzende oder umbiegende Fasern Zellen zwischen sich einschliessen, kann man nicht selten deutlich wahrnehmen, wie von Zellen, die in dem Faserzug der einen Seite liegen, Fortsätze noch eine Zeitlang in demselben Zuge bleiben und dann in einem Bogen zum andern übergehen. Dabei behalten sie entweder dieselbe Zugsrichtung bei oder gehen in die entgegengesetzte über. Anderemal, namentlich bei Durchkreuzungen, sind es vorzugsweise die von Zellen entspringenden Fasern, welche die Decussation besorgen (Fig. 5, 6). Da wo Gangliengruppen reichlich mit bindegewebigen Umhüllungen versehen sind, gehen von den Zellen nicht selten solche bindegewebige Züge zu den Nervenfasern hin, mit dem Perineurium oder Neurilem derselben sich verbindend. Diese imponiren dann wie Fortsätze, welche Ansicht aber bald durch das Fehlen des Protoplasmainhaltes, durch den deutlichen Kernreichthum des Fortsatzes vorn Beginn seines Abgangs an richtig gestellt werden kann (Fig. $7 \mathrm{~b}$ ). Eine Unterscheidung der wahren Fortsätze im engeren und weiteren Sinne war durch die unipolare Natur der Zellen von selbst ausgeschlossen. In den wenigen Fällen, in denen ich, jene Einlagerungen in den Verlauf einer Nervenfaser abgerechnet, zwei Fortsätze wahrnahm, entwickelten sich beide gleichmässig aus dem Protoplasma und verfolgten im weitern Verlaufe dieselbe Richtung. - Im Ganzen endlich findet sich für die zelligen wie für die faserigen Gebilde dieser Nervenausbreitungen der gemeinschaftliche Charakter im Bau, dass die betreffenden Theile mit Neurilem aber obne Markscheide sich darstellen. 
Ich will nun die Art der Ausbreitung der Ganglien in den Wandungen der einzelnen Stationen der Harnwege näher beschreiben. Meine Untersuchungen erstreckten sich auf die harnabführenden Organe verschiedener Thiere (Frosch, Kaninchen, Kalb, Katze, Schwein) und des Menschen und zwar bei allen von den Nierenkelchen bis zur Urethra herab. Was zunächst die Blase betrifft, so laufen die an das Organ herantretenden Nerven zunächst zugleich mit den grösseren zu- und abführenden Gefässen in die Wandungen ein, setzen unter fortgesetzten Theilungen und zugleich Verschmälerungen der Fasern neben reichlichen Anastomosen durch die Schichten derselben, um schliesslich die Mucosa zu erreichen. So kommt es, dass in den der Schleimhaut näheren Schichten und in dieser selbst nur schmalere dunkle oder blasse Nervenfasern sich darstellen und oft nur noch Stämmchen von 10-30 Primitivfasern, ja nur noch von 5-10 und noch weniger sich vorfinden, welche in die Zusammensetzung der Geflechte eingehen. Erwähnen will ich bier gleich, dass eigentliche maschenbildende Netze, welche in gleichem Niveau je in den einzelnen Schichten der Wandung ihre Lagen hätten, ich nie angetroffen habe. Es handelt sich in allen Schichten nur um das von aussen nach innen gehende Durchziehen derselhen aber verdeckt und aufgehalten durch äusserst zahlreiche Verbindungen der Nervenfaserzüge unter einander. Dabei verlaufen die einzelnen Aeste und Zweige jedoch selten längere Zeit in horizontaler Richtung, noch münden sie in geschlossene Netze, aus denen dann wieder einzelne Ausläufer in die nächsten Netzschichten ubergingen, wohl aber verlaufen sie mit ihren weiteren Verzweigungen zu den nächst oberen oder unteren, vorderen und hinteren Zügen und stellen dadurch ein Geflecht über und unter einander sich durchkreuzender und sich verbindender Nerven dar, das gleichmässig durch die ganze Dicke der Wand sich vorfindet. Einzelne dieser Schichten der Wandungen sind nur besonders reich an solchen Theilungen und Verbindungen und in solchen pflegen dann auch vorzugsweise die Ganglien eingelagert zu sein. Es finden sich aber in allen Schichten der Wandungen sowohl Anastomosen als Ganglien und es sind in den Harnorganen durchaus nicht wie im Darm diese Apparate so vorzugsweise in zwei Lager, ein submucöses und ein intermusculäres geschieden, sondern durchweg vertheilt und nur an einzelnen Stellen zahlreicher. Das entspricht 
auch den übrigen anatomischen Verhältnissen dieser Organe. An der Blase haben wir keine Schleimhaut im gewöhnlichen Sinne, kein Drüsenlager, keine Submucosa, sondern nur eine epitheltragende dichtere, mit mehr elastischen Fasern versehene, obere, und eine etwas lockere, untere Schicht, welche letztere an vielen Orten ebenfalls fehlt. Die Muscularis ist nicht in zwei überall ihre Lagerung einhaltende Schichten gesondert, sondern die circulären und longitudinalen Fasern treten nur an einzelnen Stellen, wie aussen, in gesonderten Lagen auf, im übrigen zeigen beide fortgesetzte Durchflechtungen, von welchen nur bald die eine, bald die andere das Uebergewicht hat. Die von aussen zutretenden breiten dunkelrandigen Nervenfasern beginnen sofort, wenn sie an die Wandung herantreten, ihre Theilungen und Verbindungen. Hier schon kommen grössere, selbst makroskopische Ganglien vor, ich habe dieselben aber namentlich beim Menschen nur spärlich geseben. Nach Durchsetzung der oberflächlichen Muskellage werden die Nervenfasern bald rasch dünner und in den mittleren und inneren Schichten derselben bilden meist schon nur fein dunkle oder blasse Nervenfasern die Durchflechtungen. In diesen liegen nun allenthalben die mikroskopischen Ganglien in den erwähnten mannichfachen Formen. Dabei kann man sowohl beim Menschen, als beim Thier, abgesehen von den äusseren im umhällenden Bindegewebe gelegenen Verbindungen, zwei Formen der Durchflechtungen unterscheiden. Die eine wird durch die ganze Muscularis von mittleren oder feinen dunkelrandigen und wenigen blassen Fasern gebildet, die Theilungen gescheben nicht so reichlich, die Anastomosen finden in grösseren Zwischenräumen statt, man kann hier von einem grossmaschigen Netze sprechen. Diese Nervenfaserzüge liegen in den breiteren Zwischenräumen zwischen den grösseren Muskelbündeln oder lamellenartigen Muskelausbreitungen. In diesen grösseren von Nervenverzweigungen umschlossenen Răumen findet sich eine zweite Durchflechtung ausgezeichnet von der anderen durch den Gehalt an nur feinen dunkelrandigen oder blassen Fasern, durch raschere Verzweigung und reichere Anastomosenbildung, also gleichsam ein kleineres Maschennetz innerhalb des grösseren, secundäre Durchflechtungen inverhalb der primären. Die zweiten liegen dann in den Interstitien zwischen den einzelnen kleineren und feineren Muskelbundeln. Von diesen gehen dann feinste dunkelrandige of 
varicöse oder blasse, bandartige Fasern $a b$, die sich zwischen die einzelnen Muskelzellen einsenken. Im Allgemeinen ist also auch hier ein Verhältniss, ähnlich wie Auerbach es (abgesehen von der oben berührten differenten anatomischen Anordnung der Musculatur) von dem myenterischen Nervenapparat beschrieb, wo zwischen dem inmitten der Ring- und Längsfaserlage liegenden Gangliennetzwerk ein noch feineres Nervengeflecht liegt, das aus ersterem seinen Ursprung nimmt. Klebs hat bei der Beschreibung der Nerven der Harnblasenmusculatur (Frosch) in noch eingehenderer Weise diese Nervenausbreitung gesondert und zwischen den äusseren Grundplexus und dem varicöse Fibrillen enthaltenden intermuscularen Netz noch ein, blasse, bandartige Fasern führendes, intermediäres Netz unterschieden. - Die Nervenausbreitungen in der Schleimhaut zeigen im Allgemeinen neben dunkelrandigen fast nur blasse Fasern, welche in vielfachen Verästelungen Verbindungen unter sich fast bis zur Oberfläche zieben. Aus diesen treten dann einzelne blasse bandförmige Fasern heraus, die zu dieser emporsteigen. Von diesen entzogen sich mir viele weiterer Verfolgung, andere aber konnte ich bis in das Epithel verfolgen, in welches die Faser sich einsenkte und, soviel ich sah, zwischen den Zellen frei endigte. - Was nun die Ganglien betrifft, so muss ich übereinstimmend für die Błase des Menschen wie des Thieres zunächst den Reichthum und dann die Mannichfaltigkeit dieser Gebilde betonen. Wie in der Schleimhaut des Darms, so präsentiren sich auch bei der Blase fast in jedem Objecte solche Ganglien. Sie gehören zu den grösseren dieser Art, die man zur Beobachtung bekommt und übertreffen darin selbst die des Darmes. Grosse Anhäufungen dieser Zellen, mächtige Knoten bildend, liegen in und an den Faserzügen und man begegnet nicht selten solchen, welche 20-25 und noch mehr Zellen in sich schliessen. Am reichsten finden sie sich an der Grenze zwischen Mucosa und Muscularis und nehmen von da gegen das Lumen der Blase rascher, gegen die Aussenseite des Organs langsamer in Zabl und Grösse ab. An der Grenze der Schleimhaut namentlich finden sich jene Anhäufungen sowohl in Mitte eines Faserzuges als an dessen Seite, sowohl in der Art, dass ein Faserzug von der einen Seite zutritt und mehrere auf der anderen abgehen als auch dass der Ganglienknoten gleichsam den Mittelpunkt bildet von dem strahlig nach den verschiedensten Seiten Nerven- 
faserzuge abgehen. In der Blase auch, sowohl beim Menschen als beim Thier, finden sich jene seitwärts liegenden Ganglien, die wie traubenförmige Anhänge an einem Stiele sich darstellen, welche Manz bereits schon aus der Harnblase des Frosches beschrieben hat. Sehr deutlich auch zeigen sich jene an den bogenbildenden Anastomosen vorkommenden isolirten unipolaren Zellen, welche vorzugsweise die Verbindungen zweier Nervenstämmehen mit einander besorgen. Seltener schon sind jene als einzelne oder in kleineren Gruppen auftretende Zellen in das Neurilem einer einzelnen, meist blassen Faser eingeschlossen. - Derartige Bildungen alle zeigen sich vorzugsweise in der Schleimhaut, während die in der Muscularis vorkommenden jedenfalls nicht diese Mannichfaltigkeit haben, sondern mehr uniform auftreten. Es sind das in den grösseren Maschennetzen meist in die Faserzüge eingeschlossene kleinere Knoten, gewöhnlich an den Abgangs- und Verbindungsstellen liegend. Sie bilden weniger rundliche Knoten, wie in der Schleimbaut, als längliche, mit ihrer Längsaxe mit den Muskelfaserzügen parallel laufend (Fig. 4). In den feineren und kleineren secundären Verbindungsnetzen finden sich dann neben kleinen Zellenanbäufungen zwischen den Nervenfasern jene im Verlauf von Fasern in dieselben eingebetteten, auch oft an Theilungsstellen. - Der Nachweis der wahren Fortsätze gelang bei den meisten Objecten aus den genannten $0 r-$ ganismen, ganz besonders aber beim Frosch, der Katze und dem Menschen und bei letzterem wieder besonders bei den jugendlichen Subjecten. Ich will hier gleich die weitere Wahrnehmung beifügen, dass gerade beim Menschen die Darstellung der Ganglien bei jüngeren Individuen am leichtesten gelang und dass auch die Häufigkeit des Fundes der grossen und zahlreiche schöne Zellen enthaltenden Ganglienknoten bis zum Schluss der zwanziger und dreissiger Lebensjahre zu verzeichnen ist und mit dem mittleren Alter schon abnahm, um mit zunehmendem Alter auffällig geringer zu werden. Besonders reich an Ganglien zeigte sich die Schleimhaut des Kalbes, der Katze, des Menschen aus jugendlichem Alter und auffällig reich daran fand sich andererseits die Muscularis des Schweins und der Katze.

Aehnlich wie bei der Blase findet auch an den Anfangsstücken wie an dem Endkanal der Harnleitung keine scharfe Sonderung der verschiedenen organischen Muskelschichten, sondern eine Durch- 
mischung derselben statt. Wo aber eine schärfere Trennung zu Tage tritt, findet man nicht überall, wie beim Darm, die Ringfaserschicht als die innere und darauf eine Längsschicht nach aussen, sondern oft ist letztere, wenn auch nur in schwacher Vertretung, doppelt angelegt und die Ringfaserschicht erscheint zwischen beide wie eingelagert. So findet man am Ureter schon einzelne Längsfaserschichten unter der Mucosa, mit welchen auch die Muscularis am Nierenbecken beginnt, zu denen sich dann Ringfasern gesellen, an deren äussere Seite wieder weiter abwärts am Ureter Längsschichten sich anschliessen. Vom Blasengrunde, wo die von den Ureteren kommenden Längsfasern zunächst der Mucosa liegen und nach aussen vom Sphincter vesicae internus umfasst werden, setzt sich diese Anordnung auch noch auf die Pars prostatica fort, in der Pars membranacea treten zu diesen auswärts wieder Längsfasern, die in der Pars bulbosa wieder verschwinden. Die Schleimhaut präsentirt sich in den oberen Abschnitten der Kanalwege als eine epitheltragende bindegewebige Schicht, die nur wenig elastische Fasern trägt und gleichmässig bis zur Muscularis sich erstreckt. Bei dem unteren Abschnitte lagert sich zwischen ihr und der Muscularis noch eine reichliche Gefässschicht ein.

Den Ureter mit seiner Erweiterung (Becken) umbüllt eine starke bindegewebige Schichte, welche nach innen auf der Muscularis aufliegt. Die Nerven treten als breite dunkelrandige Gebilde von aussen an den Kanal heran, um sich in fortgesetzten dichotomischen Theilungen in den Wandungen zu verbreiten. Auch hier finden sich, wie Klebs das bei der Blase des Frosches beschrieben und W. Manz es für den Ureter von Vögeln festgestellt hat, in diesen äusseren bindegewebigen Schichten an den Nerven reichliche Ganglien. Nach den Angaben von W. Manz ist hier und dann noch weiter an den äusseren Schichten der Musculatur der einzige Fundort, während in den tieferen Muskelschichten und ebenso gegen die Mucosa hin er wohl noch Nerven aber niemals Ganglien antraf. Ich hatte nicht Gelegenheit, diese Theile bei Vögeln zu untersuchen, die bewährte Forschung des Autors genügt mir aber vollständig. Für die Objecte, die icl untersuchte (Mensch, Schwein, Kalb, Kaninchen) kann ich das Vorkommen von in der äusseren Bindegewebsschicht liegenden Ganglien bestätigen, muss aber die Fundorte auch noch auf die Muscularis und die Schleimhaut erweitern. 
Der Ureter zeigt in seiner bindegewebigen Mucosa auf seinem ganzen Lauf mit seiner trichterförmigen Beckenerweiterung durchweg an seinen Nerveuverzweigungen Ganglienzellen. Sie haben zwar hier bei weitem nicht mehr jene Grösse wie bei der Blase, auch stehen sie an Häufigkeit des Vorkommens jenen nach. Die grössere Zahl liegt in den tieferen Schichten der Mucosa an der Grenze gegen die Muscularis. Sie zeigen sich vorzugsweise inmitten von Nervenfaserzügen in Gruppen von 2-3 bis 10 und noch mehr Zellen. Ich habe sie besonders schön aus der Leiche eines 10 jährigen Knaben erhalten (Fig. 14), dann aber auch beim Erwachsenen und bei allen Thieren, die ich darauf untersuchte. Der Grösse der Organe wegen, sowie in Bezug auf das Alter des Thieres ist hierzu besonders das Kalb sehr tauglich. Bei diesem lassen sich auch am deutlichsten Ganglien der Muskelschichten demonstriren. Sie liegen hier in den äusseren Partien der Längsfaserschicht und bilden dann noch weitere Gruppen an der Grenze der darauffolgenden Ringfasern. Besonders gut lässt es sich am Becken nachweisen, wo diese Schichtungen sehr deutlich hervortreten. Zwischen beiden Ganglienlagern, in der Mucoca und Muscularis, gehen zahlreicbe, meist feine, dunkelrandige Nervenfasern als Verbindungsfäden und ebenso finden sich etwas breitere, dunkelrandige Fasern, welche nach aussen mit den zuführenden und den Harnleiter umspinnenden Nerven zusammenhängen. Weiter als bis zum Nierenbecken konnte ich diese äusseren Fasern nicht verfolgen, dagegen setzten sich die in Mucosa und Muscularis befindlichen ganglientragenden Nervengeflechte in die Nierenkelche fort.

Die Spitze einer Nierenpapille trägt an ihrer Aussenseite ein niedriges Cylinderepithel, unter dem sich eine homogene oder schwach streifige Lamelle befindet, welche nach oben allmählich in eine deutliche streifige bindegewebige Unterlage ubergeht, so dass an der Umbeugungsstelle in den eigentlichen Nierenkelch schon eine deutlich entwickelte epitheltragende Mucosa sich darstellt. Von der Umschlagsstelle an treten die Verhältnisse wie beim Ureter auf, indem nach aussen von der Schleimhaut eine mit Längsfasern gemischte Ringschicht sich zeigt, an die wieder Längsfasern sich anschliessen. Wir können für uusere Zwecke die Nierenkelche in zwei Abtheilungen scheiden, eine Ganglien tragende und eine dieselben entbebrende oder auch eine mit und eine ohne Muskelschicht 
verlaufende, der die Nierenpapille überziehende Theil und der eigentliche Nierenkelch. Beide den Nierenkelch bildenden Strata, Mucosa und Muscularis, zeigen Ganglien. Sie treten aber, so weit ich hier die Verhältnisse überblicken konnte (Mensch, Kalb, Schwein, Kaninchen), nicht in Form grösserer Ganglienknoten auf, sondern bilden immer nur kleinere Gruppen, immerhin noch so, dass sie inmitten von Nervenfaserzügen eingelagert sind, aber meist in beschränkterer Zahl (bis höchstens 6-8) Zellen aufweisen (Fig. 15). Dagegen können sie sich im Verlaufe eines Nerven öfters wiederholen (Fig. 16). Dabei sind die Zellen, wie auch die des Ureters, im Ganzen kleiner als die in der Blase vorkommenden. Die Nervenfasern bilden nicht mehr so reichliche Durchflechtungen und Verbindungen und liegen nicht mehr in mebreren Etagen über einander. Sie bilden mehr oder weniger nur eine an der Grenze zwischen Muscularis und Mucosa liegende Schicht häufig anastomosirender Züge, in welchen diese Zellen eingelagert sind. Von dieser Nervenfaserschicht gehen dann äusserst zarte blasse Fasern und nur noch wenige Anastomosen bildend zur Oberfläche der Mucosa und feine dunkelrandige nach aussen in die Muscularis in die Verflechtungen dieser Schicht, von welchen dann wieder hier wie im Ureter feinste varicöse Fasern zwischen die einzelnen Muskelzellen sich einlagern.

Im unteren Abschnitt der Blase ist der Antheil der Mucosa an Ganglien ausserordentlich gross und namentlich findet sich das an den Theilen, welche über den von den Ureterenmündungen kommenden Längsfasern und an den daran anstossenden Partien des Sphincter vesicae internus liegen. Schon viel weniger zeigen sich diese Gebilde in der Schleimhaut der daran sich anschliessenden Harnröhre, wie überhaupt dieser letzte Theil der Harnwege gegenüber der Blase an Häufigkeit wie Entwicklung der Ganglien ebenso zurücktritt wie das schon von den zu diesem Reservoir fürenden Wege bemerkt worden ist. Die männliche Harnröhre zeigt entsprechend ihrem eigenthümlichen und in den einzelnen Stationen je wieder modificirten Bau eine gegenüber dem oberen Abschnitte der harnabführenden Wege wieder verschiedene Anordnung ihrer gangliösen Apparate. Es treten einmal wieder Drüsenapparate in ihr auf und dann findet sich im ganzen Verlauf der Urethra aussen an der Mucosa eine verschieden mächtige Zone von Gefässen, 
welche an einzelnen Stellen sich dem Charakter cavernöser Structur nähern. Muskelschicht und Mucosa sind daher durchweg durch eine reiche Gefässlage getrennt und auch diese und ihre sie begleitenden organischen Muskelfasern haben Ganglien (ähnlich wie die wahren Corpora cavernosa), deren Form und Anordnung ich aber ausser dem Kreise der Beschreibung lasse, da es nicht mehr streng zu den hier zu besprechenden Verhältnissen gehört. Die physiologisch postulirte Verbindung der in der Schleimbaut liegenden Ganglien mit denen der Muscularis findet daher hier nur mittelbar durch die Gefässzwischenschicht statt und es darf das wohl angenommen werden, da man von beiden Seiten her Fäden bis zur Gefässschicht ziehen sieht. - In der Schleimhaut der Pars prostatica findet sich in den tieferen Lagen gegen die Gefässschicht hin von blassen Nervenfasern gemischt mit einzelnen feinen dunkelrandigen ein Geflecht, das Ganglien trägt. Am Colliculus seminalis selbst habe ich keine gefunden, wohl aber in seiner Umgebung, namentlich zu beiden Seiten desselben. Sie enthalten in den Faserzügen eingeschlossen kleinere Gruppen von Zellen (Fig. 12). Schon hier findet sich ein Vorkommen, was in den folgenden Abschnitten immer häufiger und dann ausschliesslich wird, dass nehmlich die Zellen sich als einzelue in den Verlauf einer Nervenfaser, in deren Neurilemm, eingeschlossen finden, oft in Abständen mehrere hinter einander, so dass die Faser abwechselnd knotenförmige Anschwellung zeigt. Die Nervenfaserzüge der Muskelschicht dieses Abschnittes hängen mit denen des Blasengrundes ununterbrochen zusammen und liegen wie jene vorzugsweise nach aussen von der Längsfaserschicht, welche eine directe Fortsetzung der gleichen am Trigonon ist. An den Faserzügen sich anlegend finden sich kleine Gruppen von Zellen, welche meist von der einen Seite die Nervenfasern, von der anderen das umhällende Bindegewebe als Begrenzung haben. Sie liegen in den Interstitien $z$ wischen den grösseren Zellenbalken der Muskeln.

In der Pars membranacea sind wieder reichere Nervenausbreitungen, welche in mehrfachen Durchflechtungen die ganze Breite der Mucosa einnehmen. Aber auch hier treten die Ganglienzellen nur noch als einzelne Einlagerungen in dem Verlaufe der Nerven auf (Fig. 10, 11) und zeigen nur durch ihre grössere Häufigkeit einen Unterschied von der Pars prostatica. In der Schleimhaut der 
Pars bulbosa fand ich sie nur noch vereinzelt und nur im Anfang derselben. Die Muskelschicht der Pars membranacea zeigt zwischen der sehr mächtigen inneren Ring- und äusseren Längsfaserschicht eine ähnliche Anordnung der Ganglien wie in der der Pars prostatica. Auch hier lagern sie sich meist seitlich an die Nervenfaserzüge an. Hier wie an dem oberen Abschnitte finden sich weitere und engere Durchflechtungen und gehen von letzteren feinste varicöse oder blasse bandartige Fasern in die feinen Spalten in den Zellenbälkchen ab. Die an der Pars bulbosa allein noch übrige Ringschicht hört bald auf, so dass nun die Schleimhaut direct auf dem cavernösen Gewebe aufliegt. Ich habe in diesen Schichten Nervenfasern, immer aber nur ein und das anderemal Ganglien vorgefunden.

In der weiblichen Harnröhre dagegen sind diese Gebilde bis zum Eintritt in den Geschlechtsgang sehr deutlich entwickelt. Sowohl in der Schleimbaut dieses Ganges als in der Muskelschicht (zwei Längsfaserzüge mit mittlerer Ringschicht) kommen Gangliengruppen vor und zwar wieder grössere Anhäufungen von Zellen inmitten zahlreicher, häufige Anastomosen zeigenden Nervenfaserzüge in der Mucosa (Fig. 13) und kleinere Gangliengruppen zwischen den einzelnen Muskelschichten.

Fasst man das Gesammtresultat dieser Untersuchungen zusammen, so lässt es sich etwa folgendermaassen formuliren:

1. Ueberall durch alle Stationen der ausser der Niere gelegenen Abfubrwege des Harns beim Menschen und auch einiger Säugethiere tragen die Wandungen derselben in ihren Nervenverzweigungen Ganglien und zwar sowohl in der Mucosa als in der Muscularis.

2. In der Mucosa durchsetzen sie entweder die ganze Breite derselben oder kommen vorzugsweise oder ausschliesslich an deren Grenzpartien gegen die Muscularis vor.

3. In der Muscularis bilden die Nervenausbreitungen grössere Anastomosen in den Zwischenräumen zwischen den gröberen Muskelbalken und kleinere zwischen den feinen Muskelbälkchen dieser grösseren Schichten. Beide tragen Ganglien, die ersteren mehr.

4. Beide Nervenausbreitungen in Muscularis und Mucosa stehen in ununterbrochener Verbindung mit einander.

5. Die Nervenausbreitungen bilden uberall keine eigentlich ge- 
schlossenen Netze, sondern mehr durch bäufige Anastomosen Durchflechtungen und Verbindungen der tieferen mit den oberflächlichen Lagen.

6. Die Ganglien liegen

a) an Nervenstämmchen nur angelegt, so dass das Perineurium nur von einer Seite uber sie hinweggeht, auf der anderen Seite wird die Zellengruppe von Nervenfasern begrenzt;

b) sie liegen nicht dicht angeschlossen an die Nervenfaser in rundlicher oder spindelförmiger Gestalt, sondern länger, mehr traubenförmig an einem Stiel, ringsum von Bindegewebe umfasst;

c) die Ganglien liegen mitten in einem Nervenzug. Sie drängen dabei die Nervenfaserzuge auseinander;

d) die Ganglien liegen an Bifurcationsstellen;

e) die Ganglien liegen im Verlauf einer einzelnen Nervenfaser eingelagert.

7. Die Ganglienzellen sind, wo sie in grösserer Zahl beisammenliegen, in einem Maschenwerk des Perineurium eingeschlossen.

8. Da wo sie einzeln liegen, sind sie ganz oder theilweise von einer einfachen bindegewebigen Hülle umgeben oder auch nackt; sie sind vom Neurilem umschlossen, wenn sie im Innern von Nervenfasern sich zeigen.

9. Ein Theil der Ganglienzellen scheint apolar, ein anderer ist unipolar, wieder einige bipolar.

10. Die Fortsätze scheiden sich in wahre, d. h. wirkliche Fortsetzung des Protoplasma in eine Nervenfaser und falsche, Fortsetzung der gangliösen Hülle in die bindegewebige Hülle der Nerven.

11. Die ganglientragenden Nervenausbreitungen bestehen zum grösseren Theile aus blassen Fasern.

\section{Erklärung der Abbildungen.}

Tafel II-III.

Fig. 1. Ganglienknoten aus der Mucosa der Harnblase des erwachsenen Menschen elnen Mittelpunkt darstellend, von dem aus radiär nach verschiedenen Richtungen Faserzüge ausgehen. Hartnack 0 bj. 7, 0cul. 2. 
Fig. 2. Ganglienknoten aus der Mucosa der Harublase des erwachsenen Menschen, zu dem von links nur ein Nervenstrang zieht, rechts aber mehrere abgehen. Obj. 8, 0cul. 2.

Fig. 3. Seitlich anhångendes Ganglion aus der Mucosa der Harnblase eines Kindes. Obj. 8, 0cul. 4.

Fig. 4. Ganglienknoten zwischen gröberen Muskelbalken aus der Muscularis der Harnblase des Kaninchens. Obj. 8, 0cul. 4.

Fig. 5 a. 6. Ganglienzellen aus der Mucosa der Harnblase des Frosches. Unipolare und bipolare Zellen an den bogenförmigen Anastomosen.

Fig. 7. Ganglienzellen aus der Mucosa der Harnblase eines 12 jährigen Mädchens. a Ganglienzelle mit einem wahren Fortsatze. b Ganglienzellen nur durch Bindegewebe mit dem Nerven verbunden. Immers. 10. Ocul, 4.

Fig. 8. Ganglienzellen mit wahren Fortsätzen aus der Mucosa der Harnblase der Katze. Obj. 8, Ocul. 4.

Fig. 9. Gangllenzelle aus der Mucosa der Harnblase des Kaninchens, inmitten eines Nervenfaserzuges in ein Maschengerüst des Perineuriums eingelagert. Obj. 8, Ocul. 4.

Fig. 10 u. 11. Ganglienzellen aus der Schleimbaut der Harnröhre des Mannes (Pars membranacea). Obj. 8, 0cul. 2.

Fig. 12. Ganglienzellen aus der Mucosa der männlichen Harnröbre (Pars prostatica). a Nerven. b Gefässe. 0bj. 8, 0cul. 2.

Fig. 13. Ganglienzellen an der Grenzllnie zwischen Mucosa und Muscularis aus der Harnröhre eines 12jährigen Mädchens. 0bj. 8, 0cul. 4.

Fig. 14. Ganglienzellen in einem Nervenfaserzug aus der Schleimhaut des Ureters gegen die Muscularis zu von einem jungen Knaben. Obj. 8, 0cul. 4.

Fig. 15. Gangllenzelle aus der Muscularis der Wand eines Nierenkelches rom Kalbe. obj. 8, 0cul. 4.

Fig. 16. Ganglienzellen aus der Schleimhaut eines Nierenkelches von einem jungen Mädchen. Obj. 8, Ocul. 2. 


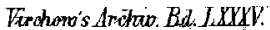

Taf.II.

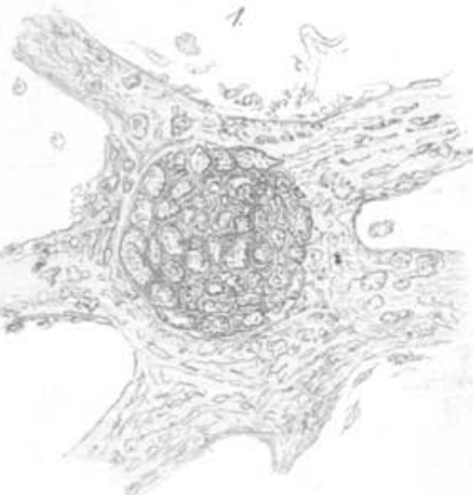

2.

3.
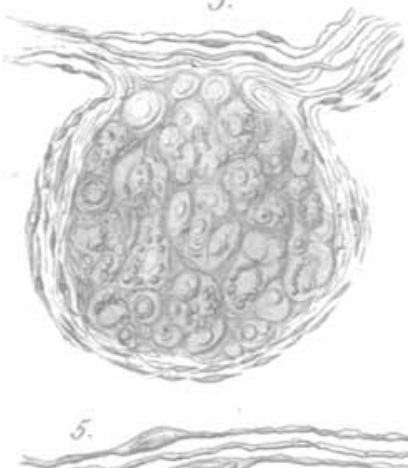

5.
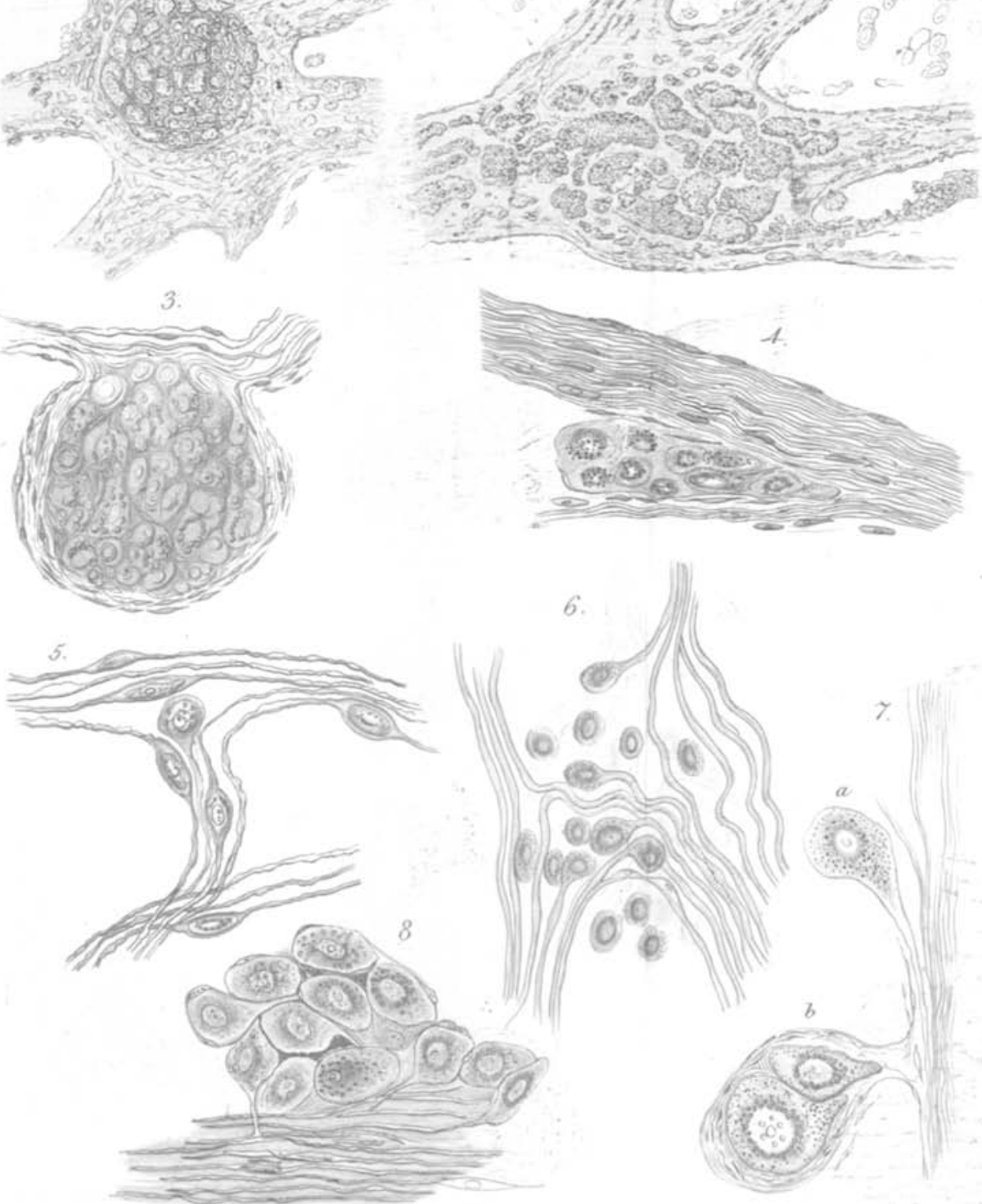

W. Grokmann as 
Vuchow's Anchu Bd. LXXXV.

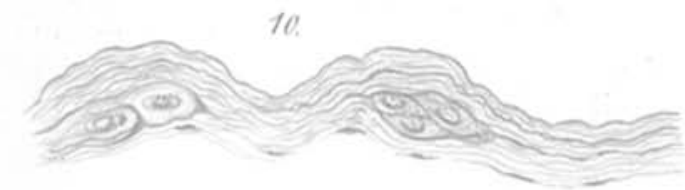

11.
Iof'III.

13.

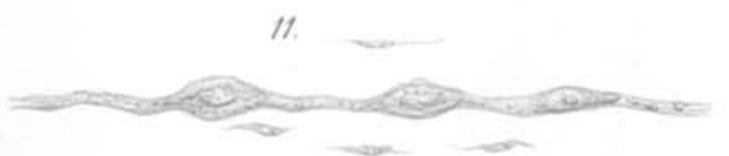

12.

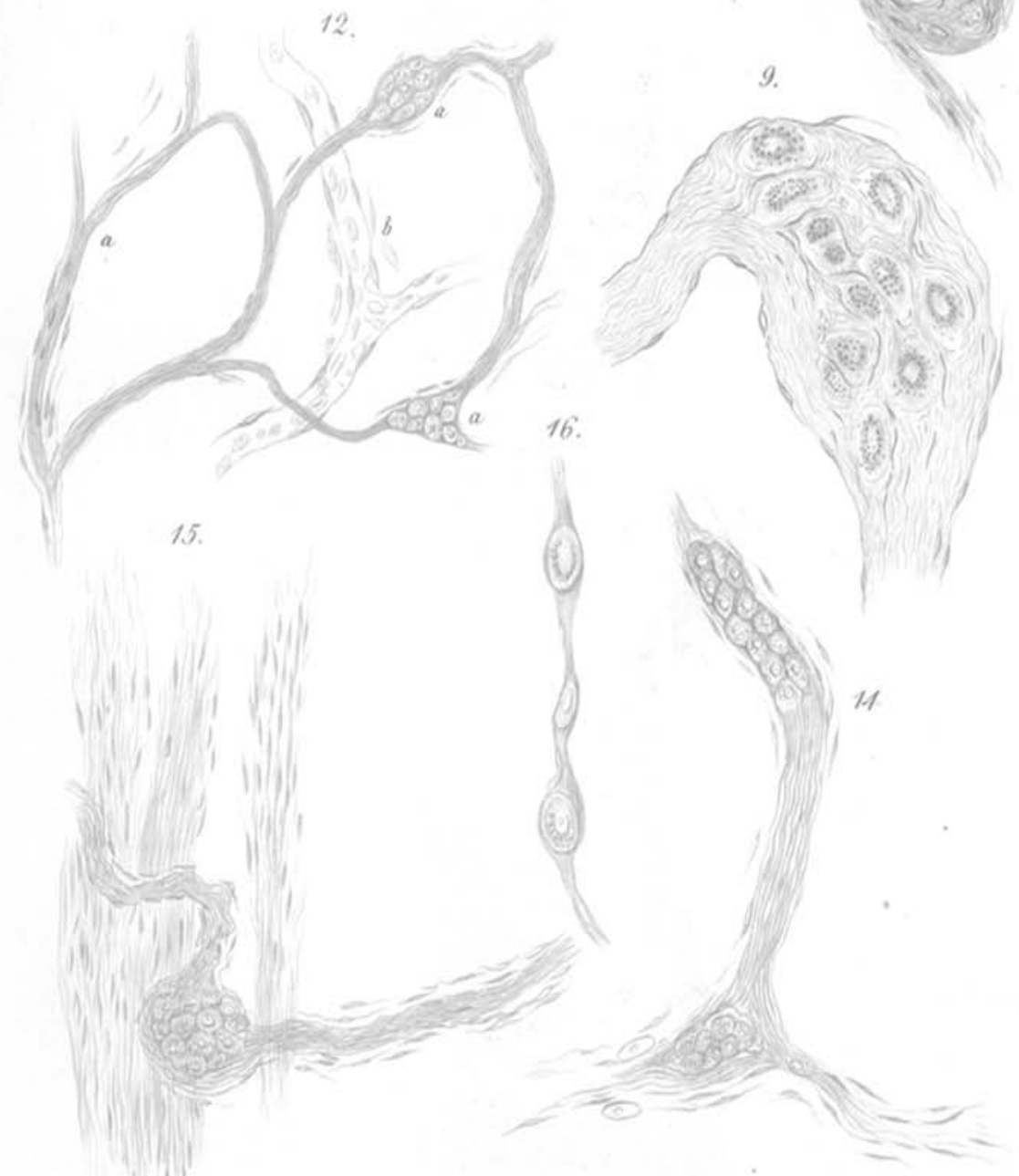

Wi Grohmans on 